\title{
A MEDIATING EFFECT OF DONGLE AND BROADBAND ON THE RELATIONSHIP BETWEEN USERS FACTORS AND CONNECTION IN LIBYA
}

\author{
Musbah .A. Musbah, Nasradeen .A. Khalifa \\ Higher Institute for Comprehensive occupations, Bani Walid Libya, Lecturer \\ tgrnna@yahoo.com \\ University Malaysia Pahang, Faculty of Engineering Technology, PhD Student \\ Nassr788@yahoo.com
}

\begin{abstract}
This is a positioning paper reflecting the problem and proposed the other name of Internet technology. It is a Global system which is interconnected with computer networks all over the world. It also uses the standard of Internet protocol suit (TCP/IP) to make a linkup with millions of computer devices globally. Internet is a web of network which interconnects millions of private, public, academic, business, and government networks, of local to global scope, that are linked by a broad array of electronic, wireless, and optical networking technologies, the aim of this study is to examining the local field data for Dongle connection and Broadband connection in for selected site and to develop a statistical relationship users and connection at selected sites as well as to validate developed a model with respect to connection at selected sites. The Internet allows greater flexibility in working hours and location, especially with the spread of unmetered high-speed connections. Internet can be accessed in anywhere by any means or medium. Internet not only be used in big screen computers or laptops but also can be used in smart phones and tablets. There are many medium or source of internet connections, but in this advance technology there are two broad sources and they are either broadband connection or dongle connection.
\end{abstract}

\section{KEYWORDS}

Computer Network, Internet Protocol, Dongle Connection, Broadband Connection, Mediation Effect.

\section{Council for Innovative Research}

Peer Review Research Publishing System

\section{Journal: INTERNATIONAL JOURNAL OF COMPUTERS \& TECHNOLOGY}

Vol. 15, No. 2

www.ijctonline.com, editorijctonline@gmail.com 


\section{INTRODUCTION}

Dongle connection is a device that attaches to a computer to control access to a particular application. Dongles provide the most effective means of copy protection. Typically, the dongle attaches to a PC's (personal computer)'s parallel port as shown below figure 1. On Macintoshes (Apple computer), the dongle sometimes attaches to the ADB (Android Debug bridge) port. The dongle passes through all data coming through the port so it does not prevent the port from being used for other purposes, as the Broadband connection is cable Internet access, shortened to cable Internet, which is a form of broadband Internet access that uses the cable television infrastructure. Like digital subscriber line and fiber to the premises services, cable Internet access provides network edge connectivity (last mile access) from the Internet service provider to an end user as shown in the figure 3. It is integrated into the cable television infrastructure analogously to DSL which uses the existing telephone network. Cable TV networks and telecommunications networks are the two predominant forms of residential Internet access. Recently, both have seen increased competition from fiber deployments, wireless, and mobile networks.
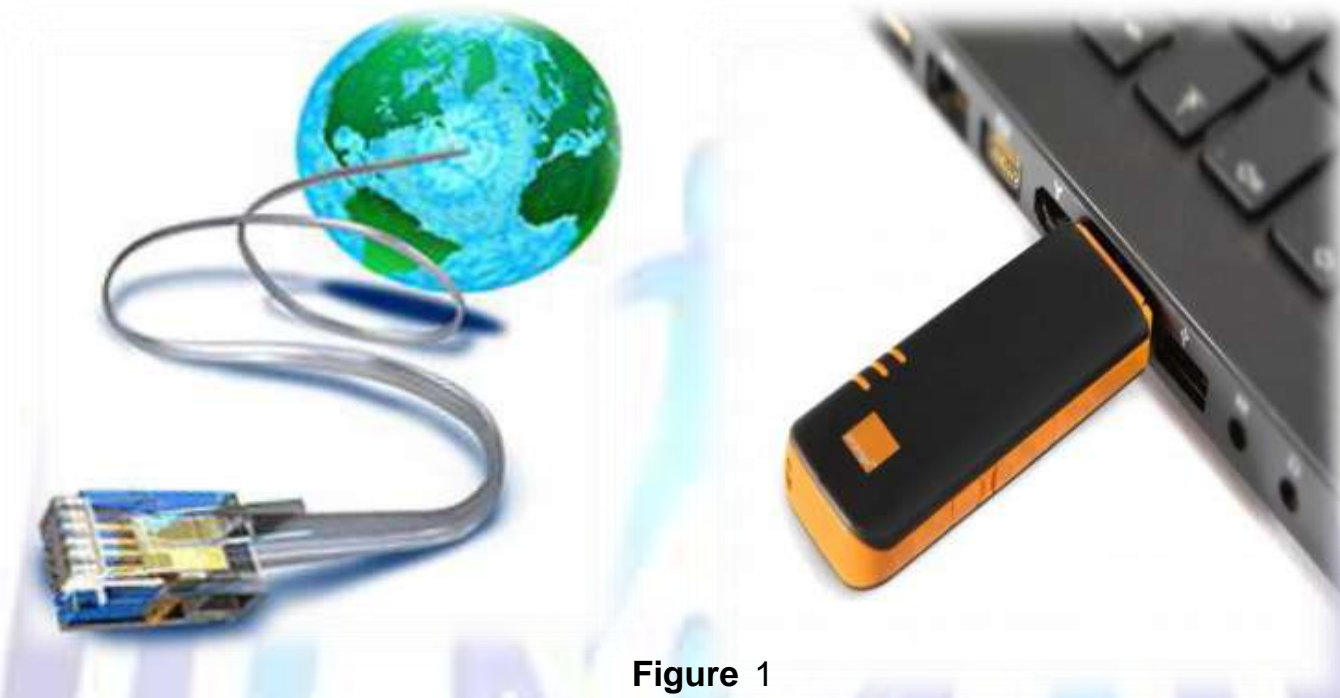

Figure 1

\section{RESEARCH PROBLEM STATEMENT}

There has been a drastic change in the mind of internet users. There are gradual shifting in their habits from any other sources to using internets with the change of technology and generations. It is found by the survey that most of the internet users are between 25 - 35 of age and the gender and the profession of the people plays a vital role in our daily life, internet helps us in every aspect. People living in Libya more price Conesus and for them it is very vital to choose a proper mode of internet connection.

\section{SIGNIFICANCE OF RESEARCH}

It is expected that this research will help the user to proper mode of internet connection, specifically the study will help improve and understanding the dongle connection and broadband connection, suggest better internet connection for other users in need. Also, the study will contribute to results positively.

\section{PRELIMINARY STUDIES}

The main, and some would say the only proper, use of the word dongle is to refer to a variety of hardware device that is used as security to safeguard proprietary software. Some use dongle, by extension, to refer to any key required for a program to operate. Some extend it in a different direction to refer to any small hardware device similar in size and shape to a dongle in the first sense and that connects to a computer port. A fourth meaning is a special adaptor cable for connecting wireless cards to an Ethernet jack. In any case, the name is said to have arisen from a variation on the word dongle, which describes how the devices hang from the computer port they are attached to.

The first kind of dongle is also known as a hardware key. Without the dongle, the software cannot be run. For many dongles, this is because the software application, upon opening, sends a code to the dongle, which is meant to respond with the serial number that unlocks the product. If this transaction is not completed, the software program is not available for use, so only an Authorized user can access the program, unless the device is lost or stolen. On other dongles, like the keylok, a portion of the executable code of the program may actually be stored on the dongle as another approach. In either case, for a user who has many licenses, the cost in lost time of a dongle being lost or stolen can be extremely high.

Early hardware keys were made for parallel ports. There are now various types of dongles that take advantage of different technologies. Examples include dongles for USB and serial ports. There are also dongles that combine hardware key function with other functions, such as flash memory. In addition Keylok has a hardware key that has networking capability and can control how many concurrent users are allowed. 


\section{VARIATIONS OF DONGLE IN LIBYAN MARKET}

Market of Libyan telecommunication is highly competitive as there exists a numerous brands. So sticking oneself to a particular connection is not a wise decision. But on the other hand the 3G data card segment in Libya is dominated by Sim locked devices.

\section{DISADVANTAGES}

- None of the $3 \mathrm{G}$ operators have a nationwide network so it may create a problem if anybody travels to a place where the same operator does not have a $3 G$ network.

- Also, with a locked SIM card, one person cannot change the operator if he is not satisfied with the service. And buying a whole new connection is generally a costly affair.

Thus, having an open network data card has a lot of advantages over locked SIM dongles. The open network data card can support any sim card of any connections and are easily available at online as well as physical retail stores. Apart, from that dongles support minimum 32 GB micro SD card as shown below in the figure 2.

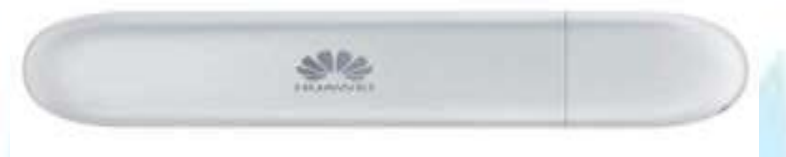

Figure 2

E303C dongle, which was launched in India for the first time, offers consumers the liberty to choose their favourite operator as per the best data plan available in the market. Customers can utilise the same SIM card used in a handset to connect and access data.

The data card offers 7.2 Mbps download speed and also works on Edge and GPRS networks. Design wise, the dongle has nothing much to offer, other than very good quality. Also, the company claims that the dongle can connect you to a network in 15 seconds even when you are using it for the first time as there is no dial process to go through.

\section{TYPES OF BROADBAND CONNECTIONS}

Broadband includes several high-speed transmission technologies such as:

- $\quad$ Digital Subscriber Line (DSL)

- Cable Modem

- Fiber

- Wireless

- Satellite

- Broadband over Power lines (BPL)

The broadband technology which people choose will depend on a number of factors. These may include whether they are located in an urban or rural area, how broadband Internet access is packaged with other services (such as voice telephone and home entertainment), price, and availability.

\section{DIGITAL SUBSCRIBER LINE (DSL)}

DSL is a wire line transmission technology that transmits data faster over traditional copper telephone lines already installed to homes and businesses. DSL-based broadband provides transmission speeds ranging from several hundred Kbps to millions of bits per second (Mbps). The availability and speed of DSL service may depend on the distance from home or business to the closest telephone company facility.

The following are types of DSL transmission technologies:

\section{- Asymmetrical Digital Subscriber Line (ADSL)}

Used primarily by residential customers, such as Internet surfers, who receive a lot of data but do not send much. ADSL typically provides faster speed in the downstream direction than the upstream direction as shown in the figure 3 . ADSL allows faster downstream data transmission over the same line used to provide voice service, without disrupting regular telephone calls on that line. 


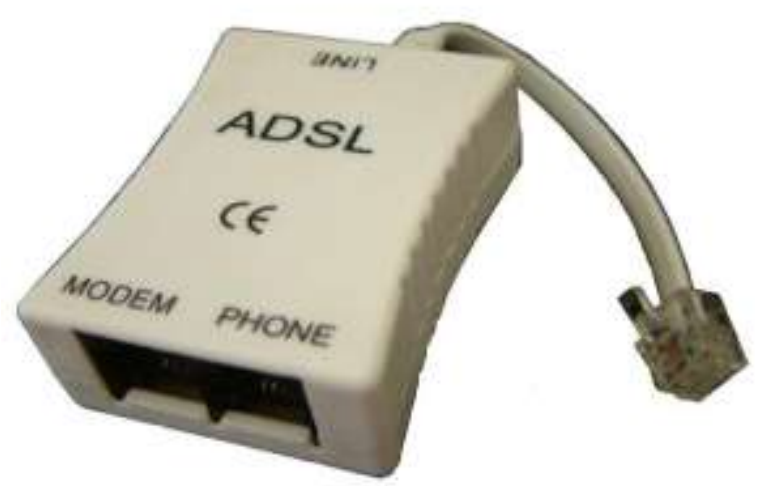

Figure 3

\section{- Symmetrical Digital Subscriber Line (SDSL)}

Used typically by businesses for services such as video conferencing, which need significant bandwidth both upstream and downstream as in the figure 4.

Faster forms of DSL typically available to businesses include:

- High data rate Digital Subscriber Line (HDSL); and

- Very High data rate Digital Subscriber Line (VDSL).

\section{CABLE MODEM}

Cable modem service enables cable operators to provide broadband using the same coaxial cables that deliver pictures and sound to your TV set.

Most cable modems are external devices that have two connections: one to the cable wall outlet, the other to a computer. They provide transmission speeds of $1.5 \mathrm{Mbps}$ or more.

Subscribers can access their cable modem service by simply turning on their computers, without dialing-up an ISP. You can still watch cable TV while using it. Transmission speeds vary depending on the type of cable modem, cable network, and traffic load. Speeds are comparable to DSL.

\section{FIBER}

- Fiber optic technology converts electrical signals carrying data to light and sends the light through transparent glass fibers about the diameter of a human hair. Fiber transmits data at speeds far exceeding current DSL or cable modem speeds, typically by tens or even hundreds of Mbps.

- The actual speed you experience will vary depending on a variety of factors, such as how close to your computer the service provider brings the fiber and how the service provider configures the service, including the amount of bandwidth used. The same fiber providing your broadband can also simultaneously deliver voice (VolP) and video services, including video-on-demand.

- Telecommunications providers sometimes offer fiber broadband in limited areas and have announced plans to expand their fiber networks and offer bundled voice, Internet access, and video services. 
- Variations of the technology run the fiber all the way to the customer's home or business, to the curb outside, or to a location somewhere between the provider's facilities and the customer.

\section{WIRELESS}

- Wireless broadband connects a home or business to the Internet using a radio link between the customer's location and the service provider's facility. Wireless broadband can be mobile or fixed.

- Wireless technologies using longer-range directional equipment provide broadband service in remote or sparsely populated areas where DSL or cable modem service would be costly to provide. Speeds are generally comparable to DSL and cable modem. An external antenna is usually required.

- Wireless broadband Internet access services offered over fixed networks allow consumers to access the Internet from a fixed point while stationary and often require a direct line-of-sight between the wireless transmitter and receiver.

- These services have been offered using both licensed spectrum and unlicensed devices. For example, thousands of small Wireless Internet Services Providers (WISPs) provide such wireless broadband at speeds of around one Mbps using unlicensed devices, often in rural areas not served by cable or wireline broadband networks.

- Wireless Local Area Networks (WLANs) provide wireless broadband access over shorter distances and are often used to extend the reach of a "last-mile" wireline or fixed wireless broadband connection within a home, building, or campus environment. Wi-Fi networks use unlicensed devices and can be designed for private access within a home or business, or be used for public Internet access at "hot spots" such as restaurants, coffee shops, hotels, airports, convention centres, and city parks.

- Mobile wireless broadband services are also becoming available from mobile telephone service providers and others. These services are generally appropriate for highly-mobile customers and require a special PC card with a built in antenna that plugs into a user's laptop computer. Generally, they provide lower speeds, in the range of several hundred Kbps.

\section{SATELLITE}

Just as satellites orbiting the earth provide necessary links for telephone and television service, they can also provide links for broadband. Satellite broadband is another form of wireless broadband, and is also useful for serving remote or sparsely populated areas.

Downstream and upstream speeds for satellite broadband depend on several factors, including the provider and service package purchased the consumer's line of sight to the orbiting satellite, and the weather. Typically a consumer can expect to receive (download) at a speed of about $500 \mathrm{Kbps}$ and send (upload) at a speed of about $80 \mathrm{Kbps}$. These speeds may be slower than DSL and cable modem, but they are about 10 times faster than the download speed with dial-up Internet access. Service can be disrupted in extreme weather conditions.

\section{BROADBAND OVER POWERLINE (BPL)}

BPL is the delivery of broadband over the existing low- and medium-voltage electric power distribution network. BPL speeds are comparable to DSL and cable modem speeds. BPL can be provided to homes using existing electrical connections and outlets. BPL is an emerging technology that is available in very limited areas as in the figure 5 . It has significant potential because power lines are installed virtually everywhere, alleviating the need to build new broadband facilities for every customer.

\section{The BPL Distribution System}

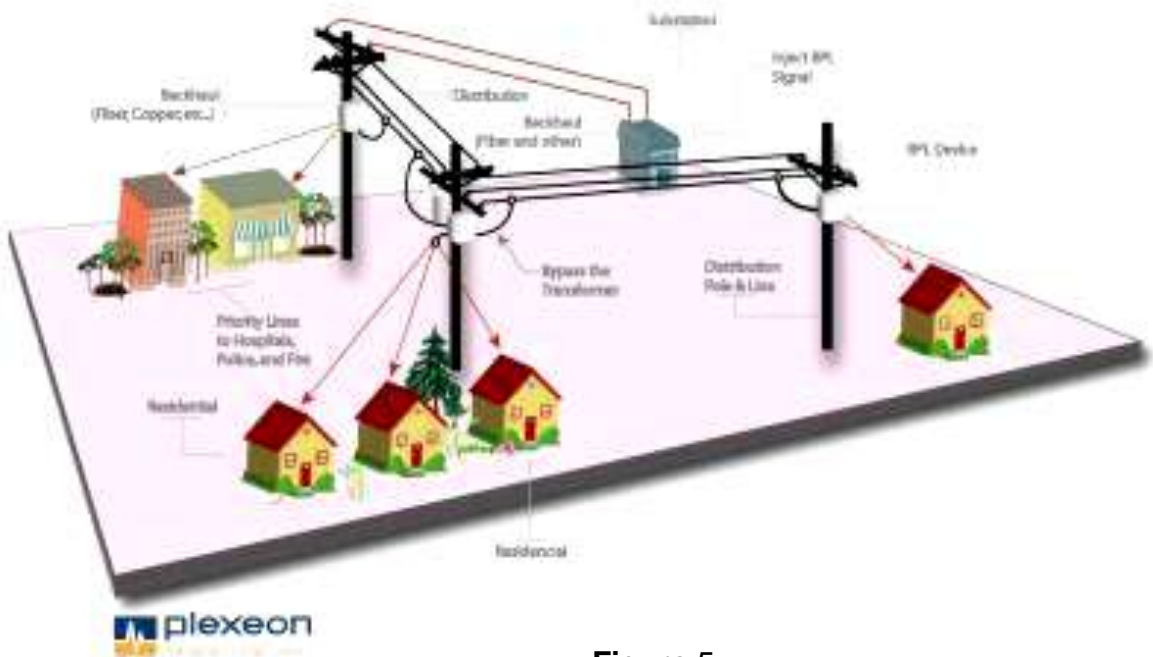

Figure 5 


\section{RESEARCH DESIGN AND METHODS}

A quantitative approach was followed. Burns and Grove (1993) define quantitative research as formal, objective, systematic process to describe and test relationships and examine cause and effect interactions among variables. Surveys may be used for descriptive, explanatory and exploratory research.

A descriptive survey design was used. A survey is used to collect original data for describing a population too large to observe directly (Mouton 1996). A survey obtains information from a sample of people by means of self-report, that is, the people respond to a series of questions posed by the investigator (Polit \& Hungler 1993). In this study the information was collected through self-administered questionnaires distributed personally to the subjects by the researcher.

A descriptive survey was selected because it provides an accurate portrayal or account of the characteristics, for example behaviour, opinions, abilities, beliefs, and knowledge of a particular individual, situation or group. This design was chosen to meet the objectives of the study, namely to determine the knowledge and views of patients and family members with regard to comparative study between dongle connection and broadband connection in Malaysia (Burns \& Grove 1993).

\section{THEORETICAL FRAMEWORK AND MODEL}

Dependent variable (DV) is area of interest, and its variable of primary interest, the variable need to be explained by independent variable of (users), and the Mediation variables of (Dongle connection and Broadband connection) and also the Dependent variable which is (area of interest), The theoretical framework identified and labelled the dependent and independent variables, the relationships among the variable will be discuss. The set of relationships that would appear in the presence of the mediation are depicted in all different models As can be seen, (Dongle connection and Broadband connection), expertise mediator the relationship between (users) and (area of interest), the mediation function of a third variable, which represents the generative mechanism through which the focal independent variable is able to influence the dependent variable of interest (Baron \& Kenny, 1986).

\section{APPROACH OF TESTING FOR MEDIATING VARIABLES}

The theory starts with the observed relationship between an independent variable $(\boldsymbol{X})$ and a dependent variable $(\boldsymbol{Y})$ such as indicated in Figure 6.

\section{MEDIATION ANALYSIS}

The role of the study is to establish the influence of independent variables on dependent variable postulating model in which intervening variable is located casually between independent and dependent variable. The intervening variable is also known as mediating variables that are conceptualized as a mechanism through which independent variables influences the dependent variable.

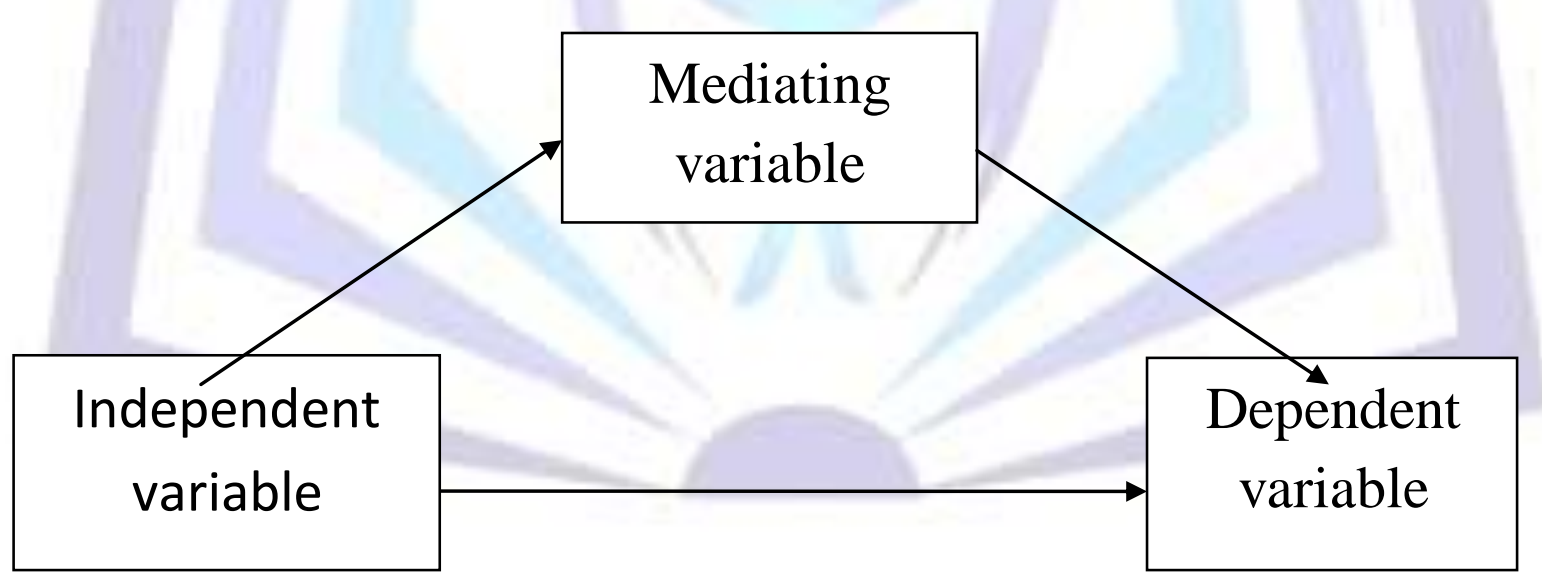

Figure 6: Mediation model with mediating variable located between independent and dependent variables

\section{LIMITATIONS}

There are several limitations that may arise in this study. The limitations may involve the objectives; samples and data collected. The first limitation is the area of focus. Since that, the general objective of this study has aimed to investigate.

\section{EXPECTED RESULTS}

The research is expected present the descriptive statistics such as the frequency distribution describes Tables, graph plots and histogram of the variables in order to get clear scenario of the data observed and to achieve objective 1 which was to examining the local field data for Dongle connection and Broadband connection in for selected site and as result of this objective the Frequency Analysis provides detail profile of categories for each variable as examination of the local field data gathered, and the Cross-Tabulation provides a cross sectional data analysis of categories between 2 of more 
than 2 variables (Hair, 2013; Sekaran, 2010; Tabachnick and Fidell, 2007), this study confirmed an achievement of research objective number 1,2 , and 3 .

\section{CONCLUSION}

This is a general theoretical framing of a mediating effect of dongle and broadband on the relationship between user's factors and connection expressing the relationship among the different factors of user's behaviour. Several sources of information should be used in order to estimate models based on this framework. This study used the questionnaire method to gather information. The modeling framework integrates these various data sources in addition to costs and environmental data to identify mediation effect that may be related to the connection. While recommendations pertaining to the Libyan government, it was acknowledged that the perspectives discussed might be applicable to all areas in general.

\section{ACKNOWLEDGMENT}

This paper was supported by the University Malaysia Pahang; and Higher Institute for Comprehensive occupations - Bani Walid Libya the authors gratefully acknowledge the support from Department of Research and innovation, UMP.

\section{REFERENCES}

[1] AL-GAHTANI, S. S. \& KING, M. 1999. Attitudes, satisfaction and usage: factors contributing to each in the acceptance of information technology. Behaviour \& Information Technology, 18, 277-297.

[2] Baron, R. M., \& Kenny, D. A. (1986). The moderator-mediator variable distinction in social psychological research: Conceptual, strategic, and statistical considerations. Journal of personality and psychology, 51 (6), 1173-1182.

[3] Marc Einstein - Frost \& Sullivan, "Dongle all the way?", Telecom Asia (21st March 2009)

[4] Erez, Eran (Gedera, IL), Carmeli, Ran (Rinatya, IL), Raines, Moshe (Tel Aviv, IL), Pomerantz, Yitzhak (KfarSaba, IL) , "Dongle joystick", G09G5/00, 11/29/2007

[5] Olivier Mardinian, "Splitter and microfilter dongle for a single RJ11 DSL/analog combo modem", (Mar 15, 2005)

[6] DAVIS, F., J.R. 1989. Perceived Usefulness, Perceived Ease of Use, and User Acceptance of Information Technology. MIS quarterly, 13, 319.

[7] DAVIS, F. D., R.P. BAGOZZI AND P.R. WARSHAW 1989. User Acceptance of Computer Technology: A Comparison of Two Theoretical Models. Management science, 35, 982

[8] HSIAO, C. H. \& YANG, C. 2011. The intellectual development of the technology acceptance model: A co-citation analysis. International Journal of Information Management, 31, 128-136.

[9] KIM, S. \& GARRISON, G. 2009. Investigating mobile wireless technology adoption: An extension of the technology acceptance model. Information Systems Frontiers, 11, 323- 333.

[10] HAM, S., KIM, W. G. \& FORSYTHE, H. W. 2008. Determinants of Restaurant Employees' Technology Use Intention: Validating Technology Acceptance Model with External Factors via Structural Equation Model Information and Communication Technologies in Tourism 2008. In: O'CONNOR, P., HÖPKEN, W. \& GRETZEL, U. (eds.). Springer Vienna.

[11] WU, W.-Y. \& LI, C.-Y. 2007. A contingency approach to incorporate human, emotional and social influence into a TAM for KM programs. Journal of Information Science, 33, 275- 297. 